PROCEEDINGS OF THE

AMERICAN MATHEMATICAL SOCIETY

Volume 137, Number 3, March 2009, Pages 1063-1068

S 0002-9939(08)09611-1

Article electronically published on October 15, 2008

\title{
A NEW PROOF OF MOK'S GENERALIZED FRANKEL CONJECTURE THEOREM
}

\author{
HUI-LING GU
}

(Communicated by Richard A. Wentworth)

\begin{abstract}
In this short paper, we will give a simple and transcendental proof for Mok's theorem of the generalized Frankel conjecture. This work is based on the maximum principle proposed by Brendle and Schoen.
\end{abstract}

\section{INTRODUCTION}

Let $M^{n}$ be an $n$-dimensional compact Kähler manifold. The famous Frankel conjecture states that: if $M$ has positive holomorphic bisectional curvature, then it is biholomorphic to the complex projective space $\mathbb{C} P^{n}$. This was independently proved by Mori 9] in 1979 and by Siu and Yau 10 in 1980 by using different methods. Mori obtained a more general result. His method was to study the deformation of a morphism from $\mathbb{C} P^{1}$ into the projective manifold $M^{n}$, while Siu and Yau used the existence result of minimal energy 2-spheres to prove the Frankel conjecture. After the work of Mori and Siu-Yau, it is natural to ask the question for the semi-positive case: what is the manifold if the holomorphic bisectional curvature is nonnegative? This is often called the generalized Frankel conjecture and was proved by Mok [8]. The exact statement is as follows:

Theorem 1.1. Let $(M, h)$ be an n-dimensional compact Kähler manifold of nonnegative holomorphic bisectional curvature and let $(\tilde{M}, \tilde{h})$ be its universal covering space. Then there exist nonnegative integers $k, N_{1}, \cdots, N_{l}$ and irreducible compact Hermitian symmetric spaces $M_{1}, \cdots, M_{p}$ of rank $\geq 2$ such that $(\tilde{M}, \tilde{h})$ is isometrically biholomorphic to

$$
\left(\mathbb{C}^{k}, g_{0}\right) \times\left(\mathbb{C} P^{N_{1}}, \theta_{1}\right) \times \cdots \times\left(\mathbb{C} P^{N_{l}}, \theta_{l}\right) \times\left(M_{1}, g_{1}\right) \times \cdots \times\left(M_{p}, g_{p}\right),
$$

where $g_{0}$ denotes the Euclidean metric on $\mathbb{C}^{k}, g_{1}, \cdots, g_{p}$ are canonical metrics on $M_{1}, \cdots, M_{p}$, and $\theta_{i}, 1 \leq i \leq l$, is a Kähler metric on $\mathbb{C} P^{N_{i}}$ carrying nonnegative holomorphic bisectional curvature.

We point out that the three-dimensional case of this result was obtained by Bando [1. In the special case, for all dimensions, when the curvature operator of

Received by the editors August 20, 2007, and, in revised form, April 5, 2008.

2000 Mathematics Subject Classification. Primary 53C20.

Key words and phrases. Generalized Frankel conjecture, holomorphic bisectional curvature, maximal principle.

The author was supported in part by NSFC 10428102 and NKBRPC 2006CB805905.

(C)2008 American Mathematical Society 
$M$ is assumed to be nonnegative, the above result was proved by Cao and Chow [4.

By using the splitting theorem of Howard-Smyth-Wu [7, one can reduce Theorem 1.1 to the proof of the following theorem:

Theorem 1.2. Let $(M, h)$ be an n-dimensional compact simply connected Kähler manifold of nonnegative holomorphic bisectional curvature such that the Ricci curvature is positive at one point. Suppose the second Betti number $b_{2}(M)=1$. Then either $M$ is biholomorphic to the complex projective space or $(M, h)$ is isometrically biholomorphic to an irreducible compact Hermitian symmetric manifold of rank $\geq 2$.

In [8, Mok proved Theorem 1.2 and hence the generalized Frankel conjecture. His method depended on Mori's theory of rational curves on Fano manifolds, so it was not completely transcendental in nature. The purpose of this paper is to give a completely transcendental proof of Theorem 1.2.

Our method is inspired by the recent breakthroughs in Ricci flow due to [2, 3]. Building upon the pioneering work of Hamilton [5], Brendle and Schoen [2] proved the differentiable sphere theorem for 1/4-pinched manifolds. Moreover in [3], the authors gave a complete classification of weakly $1 / 4$-pinched manifolds. In this paper, we will use the powerful strong maximum principle proposed in $[\underline{3}$ to give Theorem 1.2 a simple proof.

\section{The PRoof of the MAIN THEOREM}

Proof. Suppose $(M, h)$ is a compact simply connected Kähler manifold of nonnegative holomorphic bisectional curvature such that the Ricci curvature is positive at one point. We evolve the metric by the Kähler Ricci flow:

$$
\left\{\begin{array}{l}
\frac{\partial}{\partial t} g_{i \bar{j}}(x, t)=-R_{i \bar{j}}(x, t), \\
g_{i \bar{j}}(x, 0)=h_{i \bar{j}}(x) .
\end{array}\right.
$$

According to Bando [1, we know that the evolved metric $g_{i \bar{j}}(t), t \in(0, T)$, remains Kähler. Then by Proposition 1.1 in [8], we know that for $t \in(0, T), g_{i \bar{j}}(t)$ has nonnegative holomorphic bisectional curvature and positive holomorphic sectional curvature and positive Ricci curvature everywhere. Moreover, according to Hamilton [5], under the evolving orthonormal frame $\left\{e_{\alpha}\right\}$, we have

$$
\frac{\partial}{\partial t} R_{\alpha \bar{\alpha} \beta \bar{\beta}}=\triangle R_{\alpha \bar{\alpha} \beta \bar{\beta}}+\sum_{\mu, \nu}\left(R_{\alpha \bar{\alpha} \mu \bar{\nu}} R_{\nu \bar{\mu} \beta \bar{\beta}}-\left|R_{\alpha \bar{\mu} \beta \bar{\nu}}\right|^{2}+\left|R_{\alpha \bar{\beta} \mu \bar{\nu}}\right|^{2}\right) .
$$

Suppose $(M, h)$ is not locally symmetric. In the following, we want to show that $M$ is biholomorphic to the complex projective space $\mathbb{C} P^{n}$.

Since the smooth limit of a locally symmetric space is also locally symmetric, we can obtain that there exists $\delta \in(0, T)$ such that $\left(M, g_{i \bar{j}}(t)\right)$ is not locally symmetric for $t \in(0, \delta)$. Combining the Kählerity of $g_{i j}(t)$ and Berger's holonomy theorem, we know that the holonomy group $\operatorname{Hol}(g(t))=U(n)$.

Let $P=\bigcup_{p \in M}\left(T_{p}^{1,0}(M) \times T_{p}^{1,0}(M)\right)$ be the fiber bundle with the fixed metric $h$ and the fiber over $p \in M$ consisting of all 2-vectors $\{X, Y\} \subset T_{p}^{1,0}(M)$. We define a function $u$ on $P \times(0, \delta)$ by

$$
u(\{X, Y\}, t)=R(X, \bar{X}, Y, \bar{Y}),
$$


where $R$ denotes the pull-back of the curvature tensor of $g_{i \bar{j}}(t)$. Clearly we have $u \geq$ 0 , since $\left(M, g_{i \bar{j}}(t)\right)$ has nonnegative holomorphic bisectional curvature. Denote $F=$ $\{(\{X, Y\}, t) \mid u(\{X, Y\}, t)=0, X \neq 0, Y \neq 0\} \subset P \times(0, \delta)$ of all pairs $(\{X, Y\}, t)$ such that $\{X, Y\}$ has zero holomorphic bisectional curvature with respect to $g_{i \bar{j}}(t)$. Following Mok 8 , we consider the Hermitian form $H_{\alpha}(X, Y)=R\left(e_{\alpha}, \overline{e_{\alpha}}, X, \bar{Y}\right)$, for all $X, Y \in T_{p}^{1,0}(M)$ and all $p \in M$, attached to $e_{\alpha}$. Let $\left\{E_{\mu}\right\}$ be an orthonormal basis associated to the eigenvectors of $H_{\alpha}$. In the basis we have

$$
\sum_{\mu, \nu} R_{\alpha \bar{\alpha} \mu \bar{\nu}} R_{\nu \bar{\mu} \beta \bar{\beta}}=\sum_{\mu} R\left(e_{\alpha}, \overline{e_{\alpha}}, E_{\mu}, \overline{E_{\mu}}\right) R\left(E_{\mu}, \overline{E_{\mu}}, e_{\beta}, \overline{e_{\beta}}\right)
$$

and

$$
\sum_{\mu, \nu}\left|R_{\alpha \bar{\mu} \beta \bar{\nu}}\right|^{2}=\sum_{\mu, \nu}\left|R\left(e_{\alpha}, \overline{E_{\mu}}, e_{\beta}, \overline{E_{\nu}}\right)\right|^{2}
$$

First, we claim that:

$$
\sum_{\mu, \nu} R_{\alpha \bar{\alpha} \mu \bar{\nu}} R_{\nu \bar{\mu} \beta \bar{\beta}}-\sum_{\mu, \nu}\left|R_{\alpha \bar{\mu} \beta \bar{\nu}}\right|^{2} \geq c_{1} \cdot \min \left\{0, \inf _{|\xi|=1, \xi \in V} D^{2} u\left(\left\{e_{\alpha}, e_{\beta}\right\}, t\right)(\xi, \xi)\right\},
$$

for some constant $c_{1}>0$, where $V$ denotes the vertical subspaces.

The above inequality is an extension of the null-vector condition, obtained by Mok in [8, on the holomorphic bisectional curvatures (see also Hamilton's survey article [6]). More precisely, the null-vector condition of Mok [8] states that:

$$
\sum_{\mu, \nu} R_{\alpha \bar{\alpha} \mu \bar{\nu}} R_{\nu \bar{\mu} \beta \bar{\beta}}-\sum_{\mu, \nu}\left|R_{\alpha \bar{\mu} \beta \bar{\nu}}\right|^{2} \geq 0
$$

whenever $R_{\alpha \bar{\alpha} \beta \bar{\beta}}=0$. In his proof, Mok observed that if $R_{\alpha \bar{\alpha} \mu \bar{\mu}}=0$ for some vector $E_{\mu}$, then the assumption $R_{\alpha \bar{\alpha} \beta \bar{\beta}}=0$ and the associated polarization imply $R_{\alpha \bar{\mu} \beta \bar{\nu}}=0$. So in order to prove the null-vector condition, he only needed to consider the eigenvectors of $H_{\alpha}$ such that $R_{\alpha \bar{\alpha} \mu \bar{\mu}} \neq 0$. In our case, without the assumption $R_{\alpha \bar{\alpha} \beta \bar{\beta}}=0$, we cannot obtain $R_{\alpha \bar{\mu} \beta \bar{\nu}}=0$ and hence cannot apply Mok's argument in 8 directly. So we need some modification.

Inspired by Mok [8, for any given $\varepsilon_{0}>0$ and each fixed $\chi \in\{1,2, \cdots, n\}$, we consider the function

$$
\widetilde{G}_{\chi}(\varepsilon)=\left(R+\varepsilon_{0} R_{0}\right)\left(e_{\alpha}+\varepsilon E_{\chi}, \overline{e_{\alpha}+\varepsilon E_{\chi}}, e_{\beta}+\varepsilon \sum_{\mu} C_{\mu} E_{\mu}, \overline{e_{\beta}+\varepsilon \sum_{\mu} C_{\mu} E_{\mu}}\right),
$$

where $R_{0}$ is a curvature operator defined by $\left(R_{0}\right)_{i \bar{j} k \bar{l}}=g_{i \bar{j}} g_{k \bar{l}}+g_{i \bar{l}} g_{k \bar{j}}$ and $C_{\mu}$ are complex constants to be determined later. For simplicity, we denote $\widetilde{R}=R+\varepsilon_{0} R_{0}$. By setting $A_{\mu}=\widetilde{R}\left(e_{\alpha}, \overline{e_{\beta}}, E_{\mu}, \overline{E_{\chi}}\right), B_{\mu}=\widetilde{R}\left(e_{\alpha}, \overline{E_{\chi}}, e_{\beta}, \overline{E_{\mu}}\right), C_{\mu}=x_{\mu} e^{i \theta_{\mu}}(\mu \geq 1)$, where $x_{\mu}, \theta_{\mu}$ are constants to be determined later, we have:

$$
\begin{aligned}
\left.\frac{1}{2} \cdot \frac{d^{2} \widetilde{G}_{\chi}(\varepsilon)}{d \varepsilon^{2}}\right|_{\varepsilon=0}= & \widetilde{R}\left(E_{\chi}, \overline{E_{\chi}}, e_{\beta}, \overline{e_{\beta}}\right)+\sum_{\mu}\left|x_{\mu}\right|^{2} \widetilde{R}\left(e_{\alpha}, \overline{e_{\alpha}}, E_{\mu}, \overline{E_{\mu}}\right) \\
& \left.+\sum_{\mu} x_{\mu} \cdot \overline{\left(e^{i \theta_{\mu}}\left(A_{\mu}+\overline{B_{\mu}}\right)\right.}+e^{i \theta_{\mu}}\left(A_{\mu}+\overline{B_{\mu}}\right)\right) .
\end{aligned}
$$


By choosing $\theta_{\mu}$ such that $e^{i \theta_{\mu}}\left(A_{\mu}+\overline{B_{\mu}}\right)$ is real and positive and replacing $e_{\alpha}$ with $e^{i \varphi} e_{\alpha}$, the above identity becomes:

$$
\begin{aligned}
\left.\frac{1}{2} \cdot \frac{d^{2} \widetilde{F}_{\chi}(\varepsilon)}{d \varepsilon^{2}}\right|_{\varepsilon=0}= & \widetilde{R}\left(E_{\chi}, \overline{E_{\chi}}, e_{\beta}, \overline{e_{\beta}}\right)+\sum_{\mu}\left|x_{\mu}\right|^{2} \widetilde{R}\left(e_{\alpha}, \overline{e_{\alpha}}, E_{\mu}, \overline{E_{\mu}}\right) \\
& +2 \sum_{\mu} x_{\mu} \cdot\left|e^{i \varphi} A_{\mu}+e^{-i \varphi} \overline{B_{\mu}}\right|
\end{aligned}
$$

where

$$
\widetilde{F}_{\chi}(\varepsilon)=\widetilde{R}\left(e^{i \varphi} e_{\alpha}+\varepsilon E_{\chi}, \overline{e^{i \varphi} e_{\alpha}+\varepsilon E_{\chi}}, e_{\beta}+\varepsilon \sum_{\mu} C_{\mu} E_{\mu}, \overline{e_{\beta}+\varepsilon \sum_{\mu} C_{\mu} E_{\mu}}\right) .
$$

Since the curvature operators $R$ and $R_{0}$ have nonnegative and positive holomorphic bisectional curvature respectively, we know that the operator $\widetilde{R}=R_{0}+\varepsilon_{0} R_{0}$ has positive holomorphic bisectional curvature. Now choose $x_{\mu}=-\frac{\left|e^{i \varphi} A_{\mu}+e^{-i \varphi} \overline{B_{\mu}}\right|}{R\left(e_{\alpha}, \overline{e_{\alpha}}, E_{\mu}, \overline{E_{\mu}}\right)}$ (for $\mu \geq 1$ ), average the above equality over $\varphi, 0 \leq \varphi \leq 2 \pi$, and note that

$$
\widetilde{F}_{\chi}(\varepsilon)=\widetilde{R}\left(e_{\alpha}+\varepsilon e^{-i \varphi} E_{\chi}, \overline{e_{\alpha}+\varepsilon e^{-i \varphi} E_{\chi}}, e_{\beta}+\varepsilon \sum_{\mu} C_{\mu} E_{\mu}, \overline{e_{\beta}+\varepsilon \sum_{\mu} C_{\mu} E_{\mu}}\right) .
$$

Then we can obtain that

$$
\begin{aligned}
& \sum_{\mu} \widetilde{R}\left(e_{\alpha}, \overline{e_{\alpha}}, E_{\mu}, \overline{E_{\mu}}\right) \widetilde{R}\left(E_{\mu}, \overline{E_{\mu}}, e_{\beta}, \overline{e_{\beta}}\right)-\sum_{\mu, \nu}\left|\widetilde{R}\left(e_{\alpha}, \overline{E_{\mu}}, e_{\beta}, \overline{E_{\nu}}\right)\right|^{2} \\
\geq & c_{1} \cdot \min \left\{0, \inf _{|\xi|=1, \xi \in V} D^{2} \widetilde{u}\left(\left\{e_{\alpha}, e_{\beta}\right\}, t\right)(\xi, \xi)\right\},
\end{aligned}
$$

where $\widetilde{u}(\{X, Y\}, t)=\widetilde{R}(X, \bar{X}, Y, \bar{Y})=R(X, \bar{X}, Y, \bar{Y})+\varepsilon_{0} R_{0}(X, \bar{X}, Y, \bar{Y})$ and $c_{1}$ is a positive constant that does not depend on $\varepsilon_{0}$. By the arbitrariness of $\varepsilon_{0}$, we can let $\varepsilon_{0} \rightarrow 0$, and obtain that:

$$
\sum_{\mu, \nu} R_{\alpha \bar{\alpha} \mu \bar{\nu}} R_{\nu \bar{\mu} \beta \bar{\beta}}-\sum_{\mu, \nu}\left|R_{\alpha \bar{\mu} \beta \bar{\nu}}\right|^{2} \geq c_{1} \cdot \min \left\{0, \inf _{|\xi|=1, \xi \in V} D^{2} u\left(\left\{e_{\alpha}, e_{\beta}\right\}, t\right)(\xi, \xi)\right\},
$$

for some constant $c_{1}>0$. Therefore we proved our first claim.

By the definition of $u$ and the evolution equation of the holomorphic bisectional curvature, we know that

$$
\begin{aligned}
\frac{\partial}{\partial t} u(\{X, Y\}, t)= & \Delta(\{X, Y\}, t)+\sum_{\mu, \nu} R\left(X, \bar{X}, e_{\mu}, \overline{e_{\nu}}\right) R\left(e_{\nu}, \overline{e_{\mu}}, Y, \bar{Y}\right) \\
& -\sum_{\mu, \nu}\left|R\left(X, \overline{e_{\mu}}, Y, \overline{e_{\nu}}\right)\right|^{2}+\sum_{\mu, \nu}\left|R\left(X, \bar{Y}, e_{\mu}, \overline{e_{\nu}}\right)\right|^{2} .
\end{aligned}
$$

Combining the above inequality, we obtain that:

$$
\frac{\partial u}{\partial t} \geq L u+c_{1} \cdot \min \left\{0, \inf _{|\xi|=1, \xi \in V} D^{2} u(\xi, \xi)\right\},
$$

where $L$ is the horizontal Laplacian on $P$, and $V$ denotes the vertical subspaces. By Proposition 2 in [3] (actually, the same argument still holds for the bundle $P$ in [3] changed by the bundle $P$ defined in our paper), we know that the set

$$
F=\{(\{X, Y\}, t) \mid u(\{X, Y\}, t)=0, X \neq 0, Y \neq 0\} \subset P \times(0, \delta)
$$

is invariant under parallel transport.

Next, we claim that the holomorphic bisectional curvature $R_{\alpha \bar{\alpha} \beta \bar{\beta}}>0$ for all $t \in(0, \delta)$. 
Indeed, by Proposition 1.1 in [8], it is known that $g_{i \bar{j}}(t), t \in(0, \delta)$, has positive holomorphic sectional curvature, so if the claim is not true, then $R_{\alpha \bar{\alpha} \beta \bar{\beta}}=0$ for some $t \in(0, \delta)$ and some orthonormal 2 -frames $\left\{e_{\alpha}, e_{\beta}\right\}$. Therefore

$$
\left(\left\{e_{\alpha}, e_{\beta}\right\}, t\right) \in F .
$$

Combining $R_{\alpha \bar{\alpha} \beta \bar{\beta}}=0$ and the evolution equation of the curvature operator and the first variation, we can obtain that:

$$
\left\{\begin{array}{l}
\sum_{\mu, \nu}\left(R_{\alpha \bar{\alpha} \mu \bar{\nu}} R_{\nu \bar{\mu} \beta \bar{\beta}}-\left|R_{\alpha \bar{\mu} \beta \bar{\nu}}\right|^{2}\right)=0, \\
R_{\alpha \bar{\beta} \mu \bar{\nu}}=0, \quad \forall \mu, \nu, \\
R_{\alpha \bar{\alpha} \mu \bar{\beta}}=R_{\beta \bar{\beta} \mu \bar{\alpha}}=0, \quad \forall \mu .
\end{array}\right.
$$

We define an orthonormal 2-frame $\left\{\widetilde{e_{\alpha}}, \widetilde{e_{\beta}}\right\} \subset T_{p}^{1,0}(M)$ by

$$
\begin{aligned}
& \widetilde{e_{\alpha}}=\sin \theta \cdot e_{\alpha}-\cos \theta \cdot e_{\beta}, \\
& \widetilde{e_{\beta}}=\cos \theta \cdot e_{\alpha}+\sin \theta \cdot e_{\beta} .
\end{aligned}
$$

Then

$$
\begin{aligned}
& \overline{\widetilde{e_{\alpha}}}=\sin \theta \cdot \overline{e_{\alpha}}-\cos \theta \cdot \overline{e_{\beta}}, \\
& \overline{{\overline{e_{\beta}}}}=\cos \theta \cdot \overline{e_{\alpha}}+\sin \theta \cdot \overline{e_{\beta}} .
\end{aligned}
$$

Since $F$ is invariant under parallel transport and $\left(M, g_{i \bar{j}}(t)\right)$ has holonomy group $U(n)$, we obtain that

$$
\left(\left\{\widetilde{e_{\alpha}}, \widetilde{e_{\beta}}\right\}, t\right) \in F
$$

that is,

$$
R\left(\widetilde{e_{\alpha}}, \overline{\bar{e}_{\alpha}}, \widetilde{e_{\beta}}, \overline{e_{\beta}}\right)=0 .
$$

On the other hand, by the first variation and direct computations, we have

$$
R\left(\widetilde{e_{\alpha}}, \overline{\bar{e}_{\alpha}}, \widetilde{e_{\beta}}, \overline{\bar{e}_{\beta}}\right)=\cos ^{2} \theta \sin ^{2} \theta\left(R_{\alpha \bar{\alpha} \alpha \bar{\alpha}}+R_{\beta \bar{\beta} \beta \bar{\beta}}\right) \text {. }
$$

So we have $R_{\beta \bar{\beta} \beta \bar{\beta}}+R_{\alpha \bar{\alpha} \alpha \bar{\alpha}}=0$ if we choose $\theta$ such that $\cos ^{2} \theta \sin ^{2} \theta \neq 0$, and this contradicts the fact that $\left(M, g_{i \bar{j}}(t)\right)$ has positive holomorphic sectional curvature. Hence we have proved that $R_{\alpha \bar{\alpha} \beta \bar{\beta}}>0$, for all $t \in(0, \delta)$.

Therefore by the Frankel conjecture, we know that $M$ is biholomorphic to the complex projective space $\mathbb{C} P^{n}$.

This completes the proof of Theorem 1.2.

\section{ACKNOWLEDGEMEnts}

I would like to thank my advisor, Professor X.P. Zhu, and Professor B.L. Chen for their encouragement, suggestions and discussions. This paper was done under their advice. I would also like to thank the referees for their suggestions.

\section{REFERENCES}

1. S. Bando, On the classification of three-dimensional compact Kähler manifolds of nonnegative bisectional curvature, J. Diff. Geom. 19, (1984), 283-297. MR755227(86i:53042)

2. S. Brendle and R. Schoen, Manifolds with 1/4-pinched curvature are space forms, arXiv:math.DG/0705.0766 v2 May 2007.

3. S. Brendle and R. Schoen, Classification of manifolds with weakly 1/4-pinched curvatures, arXiv:math.DG/0705.3963 v1 May 2007. 
4. H. D. Cao and B. Chow, Compact Kähler manifolds with nonnegative curvature operator, Invent. Math. 83 (1986), 553-556. MR.827367 (87h:53095)

5. R. S. Hamilton, Four-manifolds with positive curvature operator, J. Differential Geom. 24 (1986), 153-179. MR862046 (87m:53055)

6. R. S. Hamilton, The formation of singularities in the Ricci flow, Surveys in Differential Geometry (Cambridge, MA, 1993), 2, 7-136, International Press, Cambridge, MA, 1995. MR 1375255 (97e:53075)

7. A. Howard, B. Smyth, and H. Wu, On compact Kähler manifolds of nonnegative bisectional curvature, I, Acta Math. 147 (1981), 51-56. MR631087 (83e:53064a)

8. N. Mok, The uniformization theorem for compact Kähler manifolds of nonnegative holomorphic bisectional curvature, J. Diff. Geom. 27 (1988), 179-214. MR925119 (89d:53115)

9. S. Mori, Projective manifolds with ample tangent bundles, Ann. of Math. (2) 110 (1979), 593-606. MR554387 (81j:14010)

10. Y. T. Siu and S. T. Yau, Complex Kähler manifolds of positive bisectional curvature, Invent. Math. 59 (1980), 189-204. MR577360 (81h:58029)

Department of Mathematics, Sun Yat-Sen University, Guangzhou, 510275 People's Republic of China

E-mail address: ghl1026@tom.com 